\title{
Collective response of self-organized clusters of mechanosensitive channels
}

\author{
Ksenia Guseva, ${ }^{1,}{ }^{*}$ Marco Thiel, ${ }^{1}$ Ian Booth, ${ }^{2}$ Samantha Miller, ${ }^{2}$ Celso Grebogi, ${ }^{1}$ and Alessandro de Moura ${ }^{1}$ \\ ${ }^{1}$ Institute for Complex Systems and Mathematical Biology, King's College, University of Aberdeen, Aberdeen AB243UE, United Kingdom \\ ${ }^{2}$ Institute of Medical Sciences, University of Aberdeen, Aberdeen AB252ZD, United Kingdom
}

(Received 12 April 2010; published 4 February 2011)

\begin{abstract}
Mechanosensitive channels are ion channels activated by membrane tension. We investigate the influence of the spatial distribution of bacterial mechanosensitive channels on activation (gating). Based on elastic short-range interactions we map this physical process onto an Ising-like model, which enables us to predict the clustering of channels and the effects of clustering on their gating. We conclude that the aggregation of channels and the consequent interactions among them leads to a global cooperative gating behavior with potentially dramatic consequences for the cell.
\end{abstract}

DOI: 10.1103/PhysRevE.83.020901

PACS number(s): 87.16.dp, 89.75.Da

Recent advances in the understanding of the functional organization of the cell membrane are shedding light on the complex dynamics of the components of the cell surface [1-3]. There is considerable evidence that nonspecific, membranemediated forces are important for the formation of protein complexes on the membrane [4]. In this work, we address the question of how the spatial organization of membrane proteins can be shaped by their short-range interactions, and how this spatial organization can affect their function. We focus on the behavior of mechanosensitive channels, which are activated by membrane deformation. These channels are present in several organisms, such as bacteria, humans, and plants, and are responsible for a variety of functions, ranging from volume regulation and locomotion to sensory input and signaling [5]. Membrane mechanical properties can also influence other types of ion channels [6]; thus mechanosensitive channels can be used as a general model for proteins actuated by membrane-mediated forces. The channels which are best characterized are from E. coli and are of two types: channels of large (MscL) and small (MscS) conductance [7]. These channels are responsible for preventing the osmotic pressure from reaching dangerous levels under hypo-osmotic shock. They are activated directly by membrane tension, which causes a membrane deformation in the channel neighborhood [8], and gate (open) when the cell is placed in an environment of low osmolarity. Although there are not many mechanosensitive channels on the E. coli membrane, they are often overexpressed for studies, and are present in larger numbers in other organisms [5]. For these reasons, they are an excellent and well-studied model system, from which broader conclusions may be drawn. Since the forces influencing them are short range, and typically attractive, there is the possibility that they form clusters. Two questions of crucial importance are, (i) Under what conditions do channels cluster? and (ii) How does clustering affect the gating of the channels? We address both questions by presenting a general statistical mechanics framework-which can be easily carried over to other types of channels with different interaction forces-and conclude that channels should indeed cluster if their density (or interaction strength) is high enough, and that this has an enormous effect

*k.guseva@abdn.ac.uk on the gating response of the channels, which display a much richer cooperative behavior than can be inferred from the characteristics of individual channels.

The collective behavior of MscL is a result of their mutual interactions, mediated by the membrane deformation around them. We model channel agglomeration and its effects on gating in a coarse-grained manner, in which the channels are placed on a two-dimensional lattice and only their nearest-neighbour interactions are considered. Initially, using a lattice gas model, we obtain the conditions for channel agglomeration, and their detailed spatial configuration. Based on that, we subsequently show that the gating dynamics of the clustered channels can be mapped onto an Ising-like model, with the addition of a spatially inhomogeneous field. This opens a new approach in the analysis of propagation of conformational states through a cluster of proteins. One of our major findings is that clustering leads to a lower threshold of channel activation, causing the clustered channels to open for lower membrane tensions than in the case of isolated channels. Furthermore, our method allows us to study nonequilibrium properties of the system such as the dynamics of transition. Due to this transition, clustering leads to an increase in the time it takes for the clustered channels to open in response to osmotic shock. Both these results show that the channel response to osmotic stress is crucially affected by interactions inside clusters.

Membrane proteins diffuse in the lipid bilayer, which can be considered as a two-dimensional fluid. They also interact with each other [1]. The possible forces among membrane proteins are electrostatic and membrane-mediated interactions [9]. The electrostatic forces can be neglected due to the charge screening effect in physiological solutions, since the Debye length is $1 \mathrm{~nm}$ [2], which is just a fraction of the size of a channel molecule. Segregation by lipid affinity is also not considered, since MscL does not exhibit strong lipid preferences in E. coli [10]. Therefore, we consider only elastic forces in what follows [9]. These forces are short range and have magnitudes of the order of $\sim k_{b} T_{0}$ ( $T_{0}$ is the typical room temperature, $T_{0}=300 \mathrm{~K}$ ). They arise from the hydrophobic mismatch between the size of the core of a protein and the length of the lipid layer that surrounds it [4]. Since lipids are more flexible than proteins, they tend to deform and adapt to the size of the protein core [see Fig. 1(a)]. It is the energy cost 


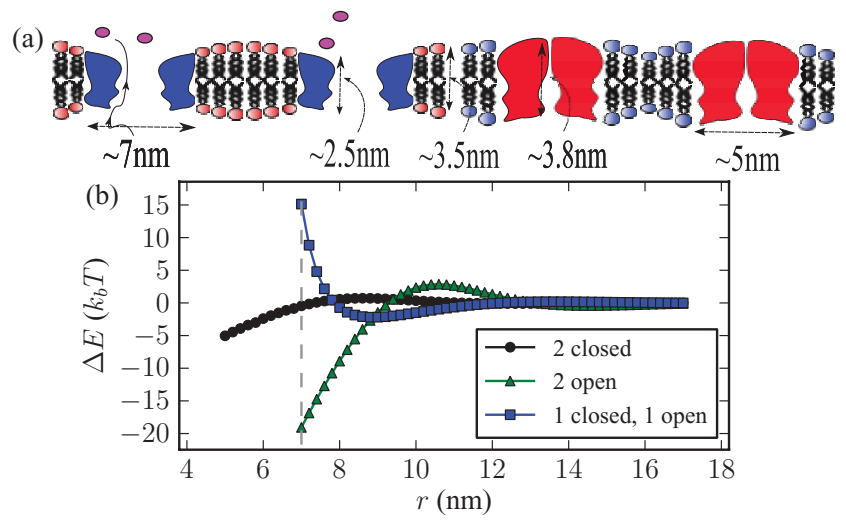

FIG. 1. (Color online) (a) Examples of the deformation profile around two open and two closed channels, left (blue) and right (red), respectively. (b) Interaction energy between two channels in different states (as in [11]).

of deforming the lipid layers which results in a force among nearby channels on the membrane. As shown in Fig. 1(a), this kind of deformation depends on whether the channel is open or closed; therefore, the forces between channels depend on their state. Since E. coli is the paradigm for such studies, we use the appropriate parameters for the MscL of these bacteria to obtain the deformation profile $\phi(r)$, defined as the distance of the membrane contour to its relaxed state, as a function of the distance $r$ to the protein. For that we minimize the free energy,

$G=\int\left[\frac{K_{a}}{2}\left(\frac{\phi(r)}{l}\right)^{2}+\frac{\kappa_{b}}{4}\left[\nabla^{2} \phi(r)-c_{0}\right]^{2}+\tau \frac{\phi(r)}{l}\right] d r^{2}$,

where $l$ is the mismatch length, the first term represents the energy cost of membrane stretching $\left(K_{a}=60 k_{b} T_{0} / \mathrm{nm}^{2}\right)$, the second is that of membrane bending $\left(\kappa_{b}=20 k_{b} T_{0}\right.$, with $c_{0}=0.009 \mathrm{~nm}^{-1}$ as the membrane curvature, we considered values in the range $0-0.04 \mathrm{~nm}^{-1}$, without any significant difference) $[8,11]$, and the last term considers the membrane tension $\tau$. To find the $\phi(r)$ which minimizes $G$, we numerically solve the Euler-Lagrange equations corresponding to Eq. (1) (see [11] for details). Considering a system of two channels, we find that the forces between them are short range $(\sim 5 \mathrm{~nm})$ [Fig. 1(b)], and they remain roughly the same for slight differences in value of $c_{0}$ and $\tau$. This distance is comparable to the diameter of a single protein, which validates our coarse-grained two-dimensional lattice approximation, which will follow.

The dynamics of agglomeration. We consider initially the normal physiological conditions for bacteria, which corresponds to a low membrane tension. In this case, all the channels are in the closed state and diffuse on the cell, interacting with each other through the elastic forces described above. Using the fact that their interaction is short range and can be approximately decomposed into pairwise interactions, we place the channels on a two-dimensional square lattice, and describe the system by the Hamiltonian $H=-J \sum_{\langle i j\rangle} s_{i} s_{j}$, where $s_{i}$ are occupancy variables associated with the lattice sites, which assume values either 1 or -1 for an occupied

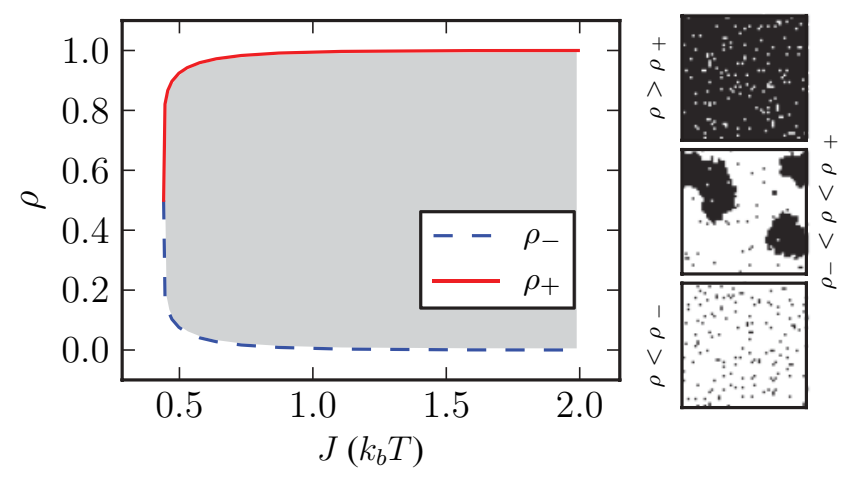

FIG. 2. (Color online) Homogeneous and nonhomogeneous state phase diagram, for $J=\frac{5 k_{b} T_{0}}{4}$ and $\rho=1.7 \times 10^{-3}$.

or free lattice site, respectively, and $J$ is the strength of the pairwise interaction between two channels. The brackets in the sum indicate that it is performed over all pairs of adjacent sites. Since the number of channels is constant, i.e., $\sum_{i} s_{i}=N \rho$, where $N$ is the total number of sites and $\rho$ is the density of occupied sites, this corresponds simply to a lattice gas, which is characterized by the existence of either a nonhomogeneous or a homogeneous phase, depending on the interaction strength $J$ (or on the thermal fluctuation $T$ ), and on the particle density $\rho$, as follows: For a weak interaction (or a high temperature) only a homogeneous phase is observed independently of the density. For $J$ high enough (or $T$ low enough) this system has two critical densities [12], $\rho_{ \pm}=\frac{1}{2}\left\{1 \pm\left[1-\operatorname{csch}^{2}(2 \beta J)\right]^{\frac{1}{8}}\right\}$. When the density $\rho$ is lower then $\rho_{-}$or higher then $\rho_{+}$ the particle distribution is homogeneous. However, if $\rho_{-}<$ $\rho<\rho_{+}$the particles segregate in different domains with different local densities: a low density $\left(\rho_{-}\right)$region and a high density $\left(\rho_{+}\right)$region (Fig. 2 ). From Eq. (1), we can determine that $J \approx 1.25 k_{b} T_{0}$, which gives $\rho_{-}=1.710^{-3}$ particles/site. Given that a wild type $E$. coli cell has on average only five channels [7] and a membrane area of $\sim 6.10^{-12} \mathrm{~m}^{2}$ [13], the density of the channels is given by $\rho \approx 1.6 \times 10^{-5}$ particles/site, which corresponds to a very low density, deep in the homogeneous phase, without any clustering. Below, when we consider the effect of agglomeration on gating, we will argue that there is a likely biological reason for this. Often, these channels are artificially over-expressed, and some authors do see nonhomogeneous distributions in such situations [14,15], suggesting that clustering may be occurring. Unfortunately they do not estimate the channel density. We suggest as a possible experiment to estimate the density in those samples and compare the appearance of agglomeration changing the density conditions.

Gating dynamics. We turn now to the gating response of the channels, when the tension is changed abruptly. This is the case, for example, if the osmolarity of the medium is suddenly decreased. We note that the gating dynamics take place on a shorter time scale than the diffusion of the channels: the gating response of channels is of the order of microseconds [16] and the free diffusion is of the order of $\sim 0.5 \mathrm{~nm}^{2} / \mu$ s for a crowded environment such as a biological membrane [17]. Since the area of a single channel is approximately $\pi(2.5)^{2} \sim 20 \mathrm{~nm}^{2}$, they cannot move significantly during a gating event, so we assume simply that their positions remain fixed in their initial 


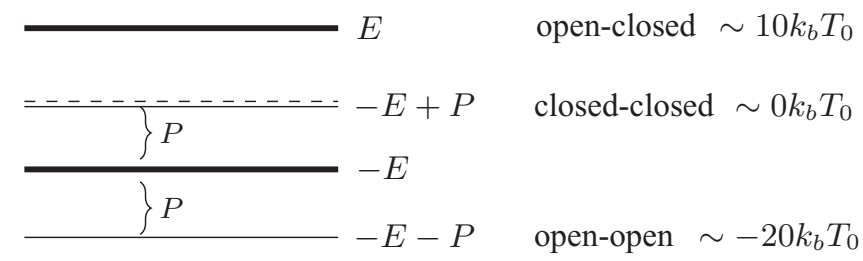

FIG. 3. Interaction energies for two channels. For $E=10 k_{b} T_{0}$ and $P=10 k_{b} T_{0}$.

values given by the lattice gas model discussed previously. Then the question is, How does the spatial clustering affect the channel's response to osmotic tension? We will describe the state of each channel $i$ by a variable $\sigma_{i}$, which can have values 1 and -1 , corresponding to an open or closed state, respectively [18]. The new energy of the system can now be written as the sum of the noninteraction energy of the individual channels and their interaction energy, $H=H_{\text {non }}+H_{\text {int }}$. These energies can be obtained by solving Eq. (1), for a system of only one or two channels, respectively, and considering all the different channel conformation states. This results in $H_{\text {non }}=h \sum_{i} \sigma_{i}$, where

$$
h=\left(\Delta G_{\text {gate }}-\tau \Delta A\right) / 2
$$

is a global noninteraction field, where $\Delta G_{\text {gate }} \sim 50 k_{b} T_{0}$ is the energy difference between conformations, $\Delta A$ is the deformation area of the protein, and $\tau$ is the the membrane tension, which changes according to the osmolarity of the medium. In an analogous fashion, we can obtain the energy levels for interacting channels, as shown in Fig. 3, for an approximate distance of around 6-7 nm from their centers. Since the interaction energies do not change significantly with tension [11], we have assumed $\tau_{0} \sim 2.5 k_{b} T_{0} / \mathrm{nm}^{2}$, which is the tension for which a single channel opens [7]. These energy levels can be written as a Hamiltonian composed of a symmetric term, plus an additional spatially correlated field, $H_{\text {int }}=-\frac{E}{2} \sum_{\langle i j\rangle} \sigma_{i} \sigma_{j}-\frac{P}{4} \sum_{\langle i j\rangle}\left(\sigma_{i}+\sigma_{j}\right)$, where the spatial correlation of the second term is due to the fact that it is summed only over neighboring sites. The second term can be interpreted as a noninteraction Hamiltonian, with particles subject to a local field $k_{i}$, which is equal to the number of occupied neighbors of site $i$, which results in $-\frac{P}{2} \sum_{i} k_{i} \sigma_{i}$. Thus, the complete energy of the system is given by the Hamiltonian

$$
H=h \sum_{i} \sigma_{i}-\frac{P}{2} \sum_{i} k_{i} \sigma_{i}-\frac{E}{2} \sum_{\langle i j\rangle} \sigma_{i} \sigma_{j},
$$

where the sums are taken over occupied sites and neighboring sites. Due to the presence of the local field $k_{i}$, this is not simply an Ising Hamiltonian. It resembles the random field Ising model [19], where the values of $k_{i}$ are replaced by randomly distributed local variables. In our case, however, they are not random, but instead they represent a quenched correlated disorder which is a byproduct of the diffusion and aggregation of the channels, as modeled by the lattice gas dynamics discussed previously.

The system given by the Hamiltonian in Eq. (3) is investigated using Monte Carlo simulations. We choose biologically relevant initial conditions in which all the particles are initialized in the closed state (mimicking conditions before an osmotic shock). This corresponds to a metastable state for the interacting particles: The transition to a global minimum (all channels open) involves the particles leaving a local minimum of the energy (all channels closed), and temporarily assuming anti-aligned states with respect to their neighbors. This process can be extremely slow, and it determines the response time of the bacterium to osmotic shock. This is potentially an important aspect of the system's dynamics: clustering can cause the channels' response time to increase. We initially examine the two extreme cases where the particle distribution is uniform. In the low-density limit $\left(\rho<\rho_{-}\right)$, most particles are isolated and there is no agglomeration. Without any mutual iteration, the individual channels will assume a preferred state given directly by Eq. (2), and will thus open when $\tau \Delta A>\Delta G_{\text {gate. }}$ The other extreme case is when the lattice is completely covered by particles, i.e., $\rho=1$ and $k_{i}=4$ for all $i$. In this case, Eq. (3) becomes the standard Ising model with external field $h-2 P$. The term $2 P$ is due to the channel interactions and, as a result, the transition to open channels occurs for a lower tension than in the case of noninteracting particles $(P=E=0)$.

For the intermediate case, when $\rho_{-}<\rho<\rho_{+}$, the process of diffusion described previously makes the channels agglomerate in clusters of finite size with highly irregular geometrical structures. These structures introduce an anisotropy in the local field $k_{i}$, which enables certain configurations of mixed states -1 and +1 to coexist. This can be observed by a comparison of the gating threshold for a group of channels inside clusters with ramified (e.g., $J=0.75$ ) or with dense (e.g., $J=1.25$ ) structures, as in Fig. 4. The transition between the two uniform global states can occur in several steps, where certain discrete groups of channels change their configuration at different values of tension. The most obvious steps correspond to the high-density regions $\left(k_{i}=4\right)$ and the low-density regions $\left(k_{i}=0\right)$, which gate for lower and for higher tensions, respectively. However, there are also intermediate transition steps that correspond to the outer layer of the clusters (particles with $1<k_{i}<4$ ), and the number of such steps varies with density. In the case of compact clusters

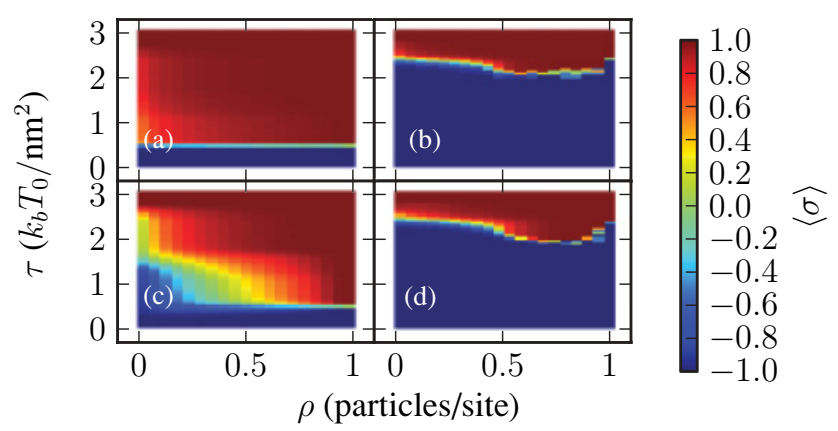

FIG. 4. (Color online) Average conformation $\langle\sigma\rangle$ as a function of particle density $\rho$ and membrane tension $\tau$. The top row corresponds to $J=1.25 K_{b} T_{0}$; bottom row to $J=0.75 K_{b} T_{0}$. (a) and (c): Equilibrium configuration. (b) and (d): Initial condition $\sigma=-1$ after a transient of $10^{5}$ iterations per particle. Simulations were carried out on a square lattice of linear size 1000 and averaged over five independent realizations. 
the transition for $k_{i}=4$ is the only one present. If we consider now a system starting from the metastable state where all channels are closed, after a finite (but large) simulation time of $10^{5}$ Monte Carlo steps per particle, the situation changes significantly, as can be seen in Figs. 4(b) and 4(d). In this case, the several discrete transition steps are replaced by a single global transition at significantly larger values of tension, characterizing a delay in the reaction time of the channels. We now turn to a more detailed comparison to the $E$. coli system. In bacteria the physiological conditions correspond to a membrane tension of $\tau \sim 0.5 k_{b} T_{0} / \mathrm{nm}^{2}$ [7]. In this condition the independent channels are all closed, since their gating threshold $\tau_{0}$ is $\sim 2.5 k_{b} T_{0} / \mathrm{nm}^{2}$. However, our theory predicts that in this situation $\tau$ is enough to trigger the gating response of clustered channels [see Fig. 4(a)]; this is a dramatic change caused by clustering on the gating dynamics [20]. However, because of the initial metastable state, clustering also increases the delay in the gating response. If long enough, this delay may be crucial, since the bacterial cell wall can only sustain high tensions for a very limited amount of time. The gating response time of a noninteracting channel is of the order of a few microseconds [16], and the survival time of the order of $100 \mathrm{~ms}$ [21]. Using the approximation derived in [22], we estimate the response time for $\rho=1$ to be $t \sim$ $\exp \frac{2 E^{2}}{k_{b} T(2 P-h)}$ Monte Carlo steps (mcs) per particle. Assuming that each mcs per particle corresponds to the characteristic reaction time of a single channel, i.e., $\sim 3 \mu \mathrm{s}$, this gives us a gating response of $\sim 20 \mathrm{~ms}$, for $\tau=\tau_{0}$. Thus we predict that under these conditions most cells would survive the osmotic shock even with clustered channels, but their gating time is orders of magnitude greater than when assuming isolated channels. This is a measurable effect with current experimental techniques, and this delay could be crucial for cells with weakened cell walls, for example. Also, even if the channels manage to open in time, the formation of compact channel clusters will cause problems for the closing of the channels (for which there will also be a delay), after the osmotic stress is removed. Clustering has therefore dramatic consequences for the nonequilibrium dynamics of the gating.

We would like to thank A. Vieira for a careful reading of the manuscript. This work was supported by the BBSRC SysMo and SABR under the Grants BB-F00513X and BB-G010722.
[1] K. Jacobson, E. D. Sheets, and R. Simson, Science 268, 1441 (1995).

[2] N. Destainville, Phys. Rev. E 77, 011905 (2008).

[3] H. Wang, N. S. Wingreen, and R. Mukhopadhyay, Phys. Rev. Lett. 101, 218101 (2008).

[4] U. Schmidt, G. Guigas, and M. Weiss, Phys. Rev. Lett. 101, 128104 (2008).

[5] P. Blount, L. Yuezhou, P. Moe, and I. Iscla, in Mechanosensitive Ion Channels (Springer, New York, 2008).

[6] E. Perozo, A. Kloda, D. M. Cortes, and B. Martinac, Nat. Struct. Mol. Biol. 9, 696 (2002).

[7] I. R. Booth, M. D. Edwards, S. Black, U. Schumann, and S. Miller, Nat. Rev. Microbiol. 5, 431 (2007).

[8] R. Phillips, T. Ursell, P. Wiggins, and P. Sens, Nature (London) 459, 379 (2009).

[9] T. Ursell, J. Kondev, D. Reeves, P. A. Wiggins, and R. Phillips, in Mechanosensitive Ion Channels (Springer, New York, 2008).

[10] A. M. Powl, J. M. East, and A. G. Lee, Biochemistry 42, 14306 (2003).

[11] T. Ursell, K. C. Huang, E. Peterson, and R. Phillips, PLoS Comput. Biol. 3, e81 (2007).
[12] M. E. J. Newman and G. T. Barkema, Monte Carlo Methods in Statistical Physics (Oxford University, New York, 1999).

[13] R. Phillips, J. Kondev, and J. Theriot, Physical Biology of the Cell (Garland Science, London, 2008).

[14] C. Norman, Z. Liu, P. Rigby, A. Raso, Y. Petrov, and B. Martinac, Eur. Biophys. J. 34, 396 (2005), PMID: 15812637.

[15] P. Wahome, A. Cowan, B. Setlow, and P. Setlow, Arch. Microbiol. 191, 403 (2009).

[16] G. Shapovalov and H. A. Lester, J. Gen. Physiol. 124, 151 (2004).

[17] S. Ramadurai, V. K. Andrea Holt, G. van den Bogaart, J. A. Killian, and B. Poolman, J. Am. Chem. Soc. 131, 12650 (2009).

[18] We emphasize that the variables $\sigma_{i}$ are unrelated to the variables $s_{i}$ used previously to describe the spatial configuration.

[19] F. Salvat-Pujol, E. Vives, and M. L. Rosinberg, Phys. Rev. E 79, 061116 (2009).

[20] This decrease in the gating threshold is consistent with previous work done with only two channels [9].

[21] M. Reutera, N. J. Hayward, S. Miller, D. T. Dryden, and I. R. Booth (unpublished).

[22] E. Cirillo and J. Lebowitz, J. Stat. Phys. 90, 211 (1998). 